\title{
Entretien avec Pierre Nora
}

Propos recueillis par Catherine Colliot-Thélène et Daniel Malbert

\section{Catherine Colliot-Thélène, Daniel Malbert et Pierre Nora}

\section{CpenEdition}

\section{Journals}

Édition électronique

URL : http://journals.openedition.org/ries/3243

DOI : $10.4000 /$ ries.3243

ISSN : 2261-4265

Éditeur

Centre international d'études pédagogiques

Édition imprimée

Date de publication : 1 mars 1997

Pagination : 53-58

ISSN : 1254-4590

Référence électronique

Catherine Colliot-Thélène, Daniel Malbert et Pierre Nora, «Entretien avec Pierre Nora », Revue internationale d'éducation de Sèvres [En ligne], 13 | 1997, mis en ligne le 01 octobre 2013, consulté le 01 mai 2019. URL : http://journals.openedition.org/ries/3243; DOI : 10.4000/ries.3243 


\section{Entretien avec Pierre Nora}

Propos recueillis par Catherine

Colliot-Thélène et Daniel Malbert

L'histoire présente en France un aspect paradoxal, que les Lieux de mémoire mettent très bien en évidence : un aspect très solide, fondé sur la continuité de l'ensemble "France » et sur un sentiment très fort d'identité nationale. Pourtant, vous avez montré en étudiant les manuels de Lavisse comment l'histoire républicaine a dû surmonter et intégrer des ruptures profondes, avant de pouvoir s'édifier. Voyez-vous là une des raisons de l'emprise du cadre national sur l'histoire?

En France, traditionnellement, les historiens ont joué un rôle idéologique d'identification très profond, ils ont dit l'identité nationale. Et le lien entre l'histoire et la nation en France remonte très haut, aux Grandes Chroniques de France, au rôle que les historiens ont pu jouer pendant les guerres de religion pour refonder l'identité française (des gens comme Étienne Pasquier dans les Recherches de la France ou La Popelinière dans l'Histoire des Français). De fait, c'est après la Révolution que l'histoire a joué un rôle particulièrement important, à cause probablement de la rupture révolutionnaire et de la nécessité de raccomoder la robe déchirée de l'histoire de la France, et puis pour toutes sortes de raisons après 1870 : selon la fameuse formule, c'est «l'instituteur qui avait vaincu à Sadowa ». Il s'agissait de refaire une armure à la patrie et l'histoire s'est présentée comme une nécessité absolue.

Alors il y a eu à la fois, en France, un surinvestissement de l'école pour des raisons qui tiennent à toute l'évolution historique : culte de la laïcité, tradition cléricale, tradition des Lumières; à l'intérieur de l'école ensuite, l'histoire a été avec le calcul et le français la discipline la plus importante de l'enseignement primaire. Dans l'enseignement supérieur, à la nouvelle Sorbonne, ce sont les historiens - comme Lavisse - qui sont apparus comme les organisateurs, les administrateurs et les concepteurs de programmes. L'histoire a véritablement été la discipline reine de la III $^{\mathrm{e}}$ République.

L'histoire de France s'est placée au centre de l'organisation quasihiérarchique de l'enseignement. D'autant qu'il s'est agi de faire de bas en haut des citoyens et des soldats. L'une des missions premières était d'acculturer un peuple peu cultivé, alphabétisé récemment afin de l'insérer à l'intérieur de l'appareil national républicain. L'éducation a été une acculturation à la vie collective et publique de la France dont l'histoire n'a été que l'évangile. D'autre part, cette mission a été très puissamment liée à l'idée de la revanche : par imitation de l'Allemagne, de sa culture, avec pour objectif de former des hommes pour qui - en cas de nécessité - la guerre serait l'instrument principal de la 
défense de la patrie. A la limite, le culte absolu de la patrie, qui a été à mille égards un substitut de la religion, dans la mesure même où la nation a été, je crois, le conservatoire d'un sacré disparu, ce culte impliquait que ses militants puissent mourir pour lui. Le sacrifice ultime de soi-même, c'est en définitive ce à quoi devait vous initier toute l'histoire de France. Si bien que cette histoire de France est devenue une grande saga unitaire, expliquant le développement national et le présentant comme un progrès permanent, aboutissant à l'apothéose de la France à travers la République et la Nation - une forme de nouvel évangile, disons.

Ce qui frappe beaucoup les étrangers, c'est l'extraordinaire unité, la cohérence $d u$ discours de l'histoire de France. Comment l'expliquez-vous?

Il y a eu une sorte d'accord profond entre la conscience nationale et la conscience historique. Cela vient, je pense, du rôle traditionnel que l'État a pu jouer en France et qu'il n'a pas joué dans d'autres pays d'une manière aussi énergique. Il n'y a qu'en France malgré tout qu'un certain nombre de grandes institutions de culture : l'école, l'université, le Collège de France, l'Académie française ont été fondées, dirigées par l'État, mais pour octroyer à ces institutions une liberté de manœuvre et une indépendance par rapport à l'État. Le rapport de l'État à la culture illustre un style dé tradition qui est très particulier en France : cette sorte de "mécénat " (terme ici transposé car en fait il conviendrait plus à l'Italie) consiste à créer et à protéger des institutions auxquelles on accorde des libertés contre vous. Je pense que le rapport à la culture et à la langue en France a été lui-même empreint d'une politique nationale très profonde, depuis le traité de Villers-Cotterêts. Et le fait que l'Académie française elle-même ait eu la fonction - symbolique, plus que réelle - de conservatoire de la langue est aussi très typique. Toutes ces institutions de culture impliquent au fond une sorte de respect de l'histoire qui a pris un aspect unitaire avec la pédagogie de la III ${ }^{\mathrm{e}}$ République. Les grands maîtres de l'université comme Lavisse en ont été les hérauts et les instruments. Ce sont les mêmes qui ont fait les manuels de l'enseignement supérieur, la Grande Histoire de France de Lavisse, et les petits manuels d'enseignement pour l'école élémentaire. On pourrait d'ailleurs sans doute relever le même phénomène en Allemagne. Cette articulation étroite entre la recherche universitaire et l'enseignement primaire ne va pas de soi: elle suppose une unité de discours historique qui n'avait pas eu lieu auparavant et qui sera rompue après. Pour que cette continuité s'accomplisse, il fallait que le fait exprime l'idée. A partir des Romantiques et jusqu'à l'histoire positiviste, la véritable révolution historienne et la science historique s'affirment par opposition à la philosophie générale, d'un côté, et, de l'autre côté, à l'accumulation du fait particulier (l'histoire "antiquaire») : désormais, c'est la nature même du fait particulier qui dégage le sens et la vérité générale. Par exemple, lorsque établir les faits de la bataille de Bouvines suffit à expliquer un des nouds de formation de la Nation elle-même. Contre l'accumulation d'un savoir érudit 
sans perspective intellectuelle et sans signification générale, contre une histoire philosophique pleine d'idées générales mais sans signification, on cherche par le récit particulier à faire ressortir la signification générale. Cela est très bien expliqué par Gabriel Monod dans le premier numéro de la Revue historique de 1867, qui est apparu comme le manifeste de cette nouvelle histoire.

Et le moule de cette articulation entre le fait particulier et l'idée générale a été l'idée nationale en France, qui lui a apposé son sceau en lui fournissant un lien de cohérence. Pour toute une génération romantique après 1820 , Augustin Thierry, Barante, Guizot, bientôt Michelet, l'histoire doit lier très étroitement l'idée nationale, la science du fait particulier, et sa signification philosophique générale. Au point qu'on pourrait dire, de manière très provocante, qu'il n'est d'histoire en France que nationale. Il faut bien sûr souligner qu'il n'y a pas que des historiens de la France, et que l'histoire n'est pas seulement nationale, mais je pense qu'à partir de 1870 et jusque vers les années 1930, l'histoire de France est restée organisatrice du champ historique.

Cette solide unité a été quand même ébranlée depuis...

Dans la crise des années 1930 s'est opérée une dissociation, dont les Annales ${ }^{1}$ ont été le signal, entre l'histoire purement nationale, assimilée à une histoire strictement politique, et la société. Les trois instances : Nation, Histoire, France se sont dissociées, dans la crise économique, dans la crise de la Nation, par le progrès et l'autonomisation des sciences sociales (l'essor de la démographie, l'étude des grandes séries économiques), qui vont rompre la continuité en montrant des évolutions parfaitement indépendantes, par exemple une France du nord et une France du sud. Alors que, dans le même temps, l'image de la Nation a cessé d'être assimilable au progrès de la civilisation, car après tout le $\mathrm{XX}^{\mathrm{e}}$ siècle n'a cessé de porter atteinte à ce mythe national (1914 et sa boucherie épouvantable, 1940 et la défaite, puis les guerres coloniales, la torture en Algérie). Tout cela va apparaître au grand jour après la guerre. Présentée longtemps comme le véhicule de la civilisation, la Nation passe bientôt pour le conservatoire des horreurs du siècle.

A vous écouter, on s'aperçoit qu'il n'y a pas que la Révolution et la défaite de 1870. Les historiens français devront-ils, selon vous, affronter de nouvelles ruptures?

Eh bien, vous pouvez constater que nous connaissons une nouvelle rupture maintenant, depuis les années 1970, qui se répercute dans l'enseigne-

1 Annales : d'après le nom de la revue Annales d'histoire économique et sociale fondée en 1929 par les historiens Lucien Febvre et Marc Bloch, qui ouvrent l'histoire aux sciences sociales et à l'étude de la longue durée. Des historiens tels que Fernand Braudel, Georges Duby ou Jacques Le Goff sont restés associés à cette démarche. Lire l'article de K. Pomian : "L'heure des Annales », dans Les lieux de mémoire, II (La Nation), tome 1, p. 377-434. 
ment. Je crois qu'on vit la troisième des grandes ruptures dans le continuum de l'unité nationale: 1820-1830, la rupture romantique; 1880 et après; la troisième rupture forte, celle d'aujourd'hui, accuse et accentue toutes les autres ruptures du siècle dont je viens de parler. Regardez la remontée de Vichy, absolument compulsionnelle, depuis une date très précise : 1971. L'affaire Touvier, la première atteinte au mythe résistancialiste, Le chagrin et la pitié, le film de Marcel Ophüls et la traduction en français du livre de Robert Paxton sur La France de Vichy. C'est tout à fait datable, entre 1971 et 1973. Et puis, c'est le début de l'après-gaullisme (de Gaulle meurt en 1970) et l'évanouissement du mythe résistancialiste qu'il avait contribué à établir. C'est aussi dans ces années 1975 que commence une très vaste transformation de la "personne nationale » sous toutes ses formes. Le début de la crise économique fait ressentir les dégâts de la croissance et la vieille France, qui s'était beaucoup mieux conservée que tous les autres pays d'Europe (souvenez-vous qu'en 1945 vous aviez encore $45 \%$ des Français aux champs !), cette France rurale est balayée en trente ans. Le succès de Montaillou d'Emmanuel Le Roy Ladurie, L'histoire de la France rurale de Georges Duby, et Le Cheval d'orgueil de Pierre Jakez Hélias vendu à un million d'exemplaires : tout d'un coup, la vieille France s'éloigne, le rural est mort. L'identité la plus traditionnelle de la France, qui est l'identité rurale, meurt et n'est plus revécue que sous la forme de livres d'histoire et de souvenirs.

Mais c'est aussi le moment où s'amorce la fin du parti communiste. L'Archipel du Goulag de Soljénitsyne paraît en 1975 et marque le début de la fin de l'idée révolutionnaire en France, très atteinte, dans ce pays qui a été traditionnellement le pays des révolutions, depuis la Révolution française. Il y a là un faisceau de transformations profondes. Le sociologue Henri Mendras emploie l'expression de "seconde révolution française " : tous les paramètres selon lesquels la France avait vécu depuis la Révolution française semblent s'épuiser. Il date le phénomène en 1965 : je crois qu'il a raison sur le plan sociologique, mais sur le plan de la conscience que les Français en ont eu, c'est à partir de 1975 que les conséquences se manifestent véritablement.

En reprenant la formule que vous appliquez à Lavisse, le professeur d'histoire est-il encore aujourd'hui un "instituteur national »? Quelles ont été les mutations dans l'enseignement à l'école?

Une des retombées dans l'enseignement, il faut le dire, a été de contribuer à défaire tous les repères de l'imagination juvénile qui consistaient dans la continuité d'un récit, l'identification à des personnages, la fixation de moments nets, de dates et de scènes archétypales où s'articulait le récit, qui perd de ce fait très largement son sens. Et les professeurs de l'enseignement secondaire - mais primaire d'abord - l'ont puissamment ressenti : ils ne savaient plus quelle histoire de France ils devaient enseigner. Fallait-il apprendre une histoire très glorieuse ? Mais ils vous répondaient : «la torture », " et les ouvriers, qui ont été exclus », " comment expliquez-vous la Commune? ?. Au moment de la guerre 
d'Algérie, la question a été particulièrement difficile : comment faire l'apologie de l'armée, quand on la savait mêlée à la torture? On pourrait d'ailleurs citer l'excellent article de Claude Billard ${ }^{2}$ qui traitait de la difficulté d'enseigner l'histoire aux enfants à l'école, en mettant en évidence pas moins de huit manières de traiter l'histoire, toutes contradictoires. Pour résumer : ou bien on continue la légende, et personne n'y croit; ou bien on fait de l'histoire de type révolutionnaire, mais en même temps ce n'est pas notre tâche de former des petits militants antipatriotes. C'était assez révélateur de dire : voilà, il y a huit manières de faire de l'histoire, on ne sait pas laquelle faire, on mélange tout et on ne sait pas du tout quoi écrire, que présenter aux enfants.

On parle beaucoup actuellement de la nécessité d'enseigner une mémoire européenne, afin de développer, de renforcer le sentiment d'identité européenne des élèves...

L'histoire européenne? Moi, je n'y crois pas. Je ne vois pas très bien quelles sont les racines d'une histoire strictement européenne. Ce serait mettre la charrue avant les bœufs, comme on dit, en présupposant un cadre qui n'existe pas encore. Et puis pensez à la question impertinente : dans une histoire strictement européenne, quelle place faites-vous à Jeanne d'Arc, anti-anglaise? En revanche, la seule histoire de type européen qu'on puisse inculquer civiquement, c'est une histoire démocratique, une histoire des valeurs de la démocratie au sens large du mot. Cela dit, elle est aussi américaine qu'européenne. Mais sur le fond, oui, il n'y a pas de doute qu'on peut expliquer aux enfants l'état de droit, le refus du terrorrisme, les libertés individuelles, la liberté de se déplacer sans contrôle, la liberté de la personne : ce sont des idées qu'on peut expliquer concrètement et facilement. Mais encore une fois, est-ce une idée strictement européenne ? Et la colonisation, faut-il expliquer que c'est un phénomène strictement européen, aussi ?

Justement, les Lieux de mémoire, dans leur démarche même, semblent typiquement français et destinés à une sorte d'introspection de l'identité nationale...

Il y a deux choses qu'il faut dire à propos des Lieux de mémoire. La première est qu'il est évident que c'est une histoire plus proche du citoyen que l'histoire économique et sociale (que je ne confonds pas avec l'histoire issue des Annales, qui justement ne s'est jamais réduite à l'analyse économique et sociale). Une histoire qui parle de choses que tout le monde connaît: la Tour Eiffel, le 14 juillet, la devise «Liberté, Égalité, Fraternité ». Tout ce qui ressortit à la mémoire est par nature plus proche du vécu spontané des gens. D’autre part, j'ai cru fermement, en lançant cette entreprise, que la notion était particulièrement adaptée à la France en fonction de tout ce que je rappelais de cette histoire

2 Claude Billard, "Enseigner l'histoire », Le Débat n 16, Paris, Gallimard. 
si unitaire, si cristallisée, si essentielle à l'identité nationale, plongeant ses racines très loin, avec même cette ambiguïté d'origine entre Francs et Gaulois. En cela, je me suis carrément trompé et j'ai été très surpris de voir que la notion rebondit à l'étranger - au point même d'être copiée récemment en Italie de manière très discutable - et qu'on dénombre à l'heure actuelle quantité de projets en Allemagne, en Espagne, aux États-Unis, en Russie, en Israël... En comparant ce qui se passe dans les différents pays, j'ai l'impression que nous voyons émerger une loi de la mémoire qui se manifeste par une vague mémorielle de fond.

\section{Ouvrages cités}

NORA Pierre (dir.), Les lieux de mémoire, I La République (1984); II La Nation (3 vol., 1986) ; III Les France (3 vol., 1993) ; Paris, Gallimard. PAXTON Robert, La France de Vichy, 1940-1944, Paris, Seuil, "coll. Points Histoire ", 1973.

ROUSSO Henry, Le syndrome de Vichy de 1944 à nos jours, Paris, Seuil, 1990 ( $2^{e}$ édition).

CONAN Eric et ROUSSO Henry, Vichy. Un passé qui ne passe pas, Paris, Fayard, 1994.

JAKEZ HÉLIAS Pierre, Le Cheval d'orgueil, Paris, Plon, 1975.

LE ROY LADURIE Emmanuel, Montaillou, village occitan, Paris, Gallimard, 1982.

DUBY Georges (dir.), Histoire de la France rurale, Paris, Seuil, 1992, 4 vol. MENDRAS Henri, La Seconde Révolution française, 1965-1984, Paris, Gallimard, "coll. Folio-Essais », 1994. 